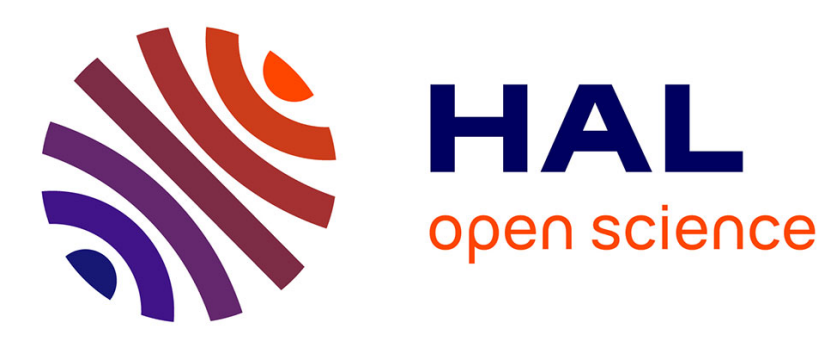

\title{
A Socio-cognitive Approach to Personality: Machine-learned Game Strategies as Cues of Regulatory Focus
}

Caroline Faur, Philippe Caillou, Jean-Claude Martin, Celine Clavel

\section{- To cite this version:}

Caroline Faur, Philippe Caillou, Jean-Claude Martin, Celine Clavel. A Socio-cognitive Approach to Personality: Machine-learned Game Strategies as Cues of Regulatory Focus. Affective Computing and Intelligent Interaction (ACII 2015), Sep 2015, Xi'an, China. hal-01216540

\author{
HAL Id: hal-01216540 \\ https://hal.inria.fr/hal-01216540
}

Submitted on 29 Jan 2016

HAL is a multi-disciplinary open access archive for the deposit and dissemination of scientific research documents, whether they are published or not. The documents may come from teaching and research institutions in France or abroad, or from public or private research centers.
L'archive ouverte pluridisciplinaire HAL, est destinée au dépôt et à la diffusion de documents scientifiques de niveau recherche, publiés ou non, émanant des établissements d'enseignement et de recherche français ou étrangers, des laboratoires publics ou privés. 


\title{
A Socio-cognitive Approach to Personality: Machine-learned Game Strategies as Cues of Regulatory Focus
}

\author{
Caroline Faur*, Philippe Caillou ${ }^{\dagger}$, Jean-Claude Martin*, Celine Clavel ${ }^{*}$ \\ *LIMSI-CNRS, Orsay, France \\ Email: \{faur, martin, clavel\}@limsi.fr \\ ${ }^{\dagger}$ LRI, Orsay, France \\ Email: caillou@1ri.fr
}

\begin{abstract}
Artificial agents are becoming artificial companions, interacting with the user on a long-term basis. This evolution brought new challenges to the affective computing domain, such as designing artificial agents with personalities to the benefits of the user. Endowing artificial agents with personality could help to increase the agent's believability, hence easing the interaction. This paper touches on two questions pertaining to computational personality modeling: 1/ how to produce artificial personalities which can inform personality researchers, whether from computer sciences or psychology and 2/ will behaviors produced by artificial agents be perceived by users as putting the programmed personality across as such. We propose to use a data-driven approach to endow artificial agents with personality, using the regulatory focus theory as a framework. We used machinelearned game strategies, in the form of alternative decision trees computed from human data, to convey the personality of artificial agents. We then tested whether these personalities can be perceived by users after playing a game against these agents. We used two artificial agents as controls: one randomly playing and one with an "average / depersonalized" strategy. On the one hand, our results show that agents' regulatory focus, when programmed, can be accurately perceived by users. On the other hand, our results also point out that personality will be perceived by users even if the agent's design does not intend to transmit one.
\end{abstract}

\section{INTRODUCTION}

In the last decade, software agents were brought to a new level, due to technological evolutions : artificial agents ceased to be only human-computer interfaces to become artificial companions [1]. On the one hand, an artificial companion can be defined as "a personalised, multi-modal, helpful, collaborative, conversational, learning, social, emotional, cognitive and persistent computer agent that knows its owner, interacts with the user over a long period of time and builds a (long-term) relationship to the user" [2]. On the other hand, personality can be defined as a coherent patterning of affect, behavior, cognition, and desires (goals) over time and space [3]. Knowing that credibility (i.e. the capacity of being perceived as believable and convincing [4]) is assessed by the consistency and the coherence of an artificial entity at various levels (psychological and physical; intrapersonal as well as social [5], [6]), endowing artificial companions with personality could help to increase the companion's believability, hence easing the interaction and, thereby, producing an adequate environment for a "relationship" to take place.

Now the question is: how to endow artificial entities with personality? Following Vinciarelli and Mohammadi [7], we like to think of personality as "a common ground where multiple disciplines, including computing and psychology, can contribute and mutually benefit from each other: progress in personality theory should help to build more effective personality machines and vice versa". So, turning to personality psychology, computer scientists have found two principal types of models: traits models and socio-cognitive models. Both have been used in affective computing, the former more than the latter. Yet, socio-cognitive models, which are interested in structures and processes of personality, provide an interesting background to computer scientists. With interpretable computational models relying on socio-cognitive theories, affective computing can inform psychologists on the "validity" of these ones or, at least, their capacity to represent a process resulting in consistent behaviors. If implementing artificial personalities is an open research question, perceiving personality should not be an issue for the user: people can find personality in many things, from moving geometrical shapes [8] to their own cars [9]. But may users perceive the intended personality chosen by programmers for their artificial companions?

To try to answer these questions, we adopt the following process: 1/ run a experiment to collect human data; $2 /$ use a classifier to determine the important features guiding human's behaviors; 3/ design artificial agents based on the result of the classification; 4/ set a user study to verify the users' perception of agents' personalities. Most of the works in affective computing are theory-driven. Theory-driven models transform the (mostly qualitative) knowledge provided by psychological models into an implementation, symbolic or not, by a deductive logic. The issue lies in the gap between the constraints of implementation and the generality of the psychological models used. That is why we propose to take a data-driven approach, i.e. our implementation will try to produce behaviors as close as possible to empirical data by using classification methods which could produce interpretable outputs. That way, it may be possible to use an inductive logic 
to infer more theoretical mechanims and yet, our results could feed future symbolic implementation.

In this article, we start with related works in the field of psychology and affective computing (Section II). Further, we will present our data-driven modelisation of personality, based on the regulatory focus theory, for an artificial agent playing a board game with a user (Section III), along with the user study about perception of the agent's personality and credibility (Section III-D). Then, we present and discuss the results of this study (Section IV) and finally, we conclude and propose future directions (Section V).

\section{RELATED WORK}

\section{A. Personality in psychology and affective computing}

In 2000, Nass and Moon [10] suggest the Computers As Social Actors (CASA) paradigm. The CASA paradigm states that people tend to adopt social attitudes with machines that can elicit social heuristics. Personality can be attributed by users to a computer and have an influence over users' behaviors. In this view, designing specific personalities that are perceived such as they were designed seems especially important for any computer scientist taking interest in artificial personality. If computer scientists make sometimes their own model of personality [11], most of the works in affective computing lean on psychological models, such as the Five Factors Model (FFM) [12] which defines five traits of personality. Because FFM is a dominant and well-known model in personality psychology, this model is naturally becoming the most used reference in affective computing [13]. The majority of FFM-inspired models use symbolic implementation but some propose a network approach, such as [14] which create a neural network based on 240 items of the NEO-PI-R (a FFM questionnaire for assessing traits of personality) and suggest that the stability of personality comes from the stability of this network structure. But FFM and other traits theories propose a descriptive approach of personality. Thus, this kind of theories helps to grasp "what" is personality but cannot guide computer scientists on the "how": how to link behaviors to personality. On the contrary, socio-cognitive models are explicative per se.

The socio-cognitive approach to personality attempts to understand cognitive and social processes that lead to personality and underlines the importance of a situation in exhibiting personality behaviors [15]. For that purpose, it focuses on the interaction between the person and the social context and highlights the intra-individual differences [16]. There are few works taking a socio-cognitive approach in affective computing. Among them, it is not uncommon to find machinelearning mechanisms and especially neural networks models: the SPOT (Simulating Personality Over Time) model combines the five traits of FFM with situational factors inside a neural network which determine output behaviors of virtual agents [17]; the BIS/BAS theory (Behavioral Inhibition System / Behavioral Approach System) has been operationalized in a neural network model of personality which links situational features, resources and motivations [18]. After training, simulations showed this network's ability to produce stable behavioral signatures (i.e. consistent associations between situations and behaviors). Nonetheless, neural network approaches have a major drawback: unlike symbolic implementation, it lacks explanatory power when trying to understand personality processes [19].

\section{B. Using the regulatory focus theory}

To try to answer our first question - how to endow artificial entities with personality? - we propose to take a socio-cognitive perspective by using the regulatory focus as a framework, as suggested by [20]. The regulatory focus theory is of interest here because it provides insights about how different personalities can be represented as functions of different processes.

The regulatory focus theory [21] distinguishes between two self-regulation strategies: 1/ promotion-focus, look into with the presence or absence of positive outcomes, gains versus nongains and 2/ prevention-focus, look into with the presence or absence of negative outcomes, losses versus non-losses. According to this theory, promotion-focus people would be more prone to using their ideal selves as guides for their behaviors (i.e., they are looking for being what they want to be) than prevention-focus people, who would prefer using ought selves (i.e., they are looking for being what they think they have to be). Promotion and prevention are two independent dimensions. One person has both a promotion-focus and a prevention-focus score. Regulatory focus can be situational, i.e. induced by context, but theory states that people have a chronic focus, i.e. an "habitual" focus used by default.

\section{Conveying personality via game strategies}

As an application, we selected strategy during a game as the first and only modality for expressing the artificial agent's personality. Several links have been made between personality and games, in psychology ([22], [23]) and in computer sciences ([24], [25]). Games are quite relevant for designing and evaluating affective agents ([26], [27]). We selected a board game, named "Can't Stop" (designed by Sid Sackson [28]). At each turn of this game, the player has to choose between either stopping a turn, i.e. saving the current gains but losing in speed, or playing again, i.e. taking the risk of losing the current gains to win more. We selected this very game because it enables to study strategies in terms of promotion and prevention since at each turn the player has to $1 /$ select a movement that can be more or less risky and $2 /$ make a choice between a vigilant strategy (stopping) or an eager strategy (playing again).

\section{DESIGNING ARTIFICIAL AGENTS WITH A REGULATORY FOCUS PERSONALITY}

We designed artificial agents playing a board game in which strategy can convey the agent's focus. In order to have data to compute machine-learned data-driven strategies for our agents, we choose to record human-vs-human game sessions. With the self-assessment of participants' regulatory focus, the games' data can be used to learn the strategies set by human players 
and test whether these strategies are influenced by the player's personality. Our methodology is composed of the following steps: 1/ learning human strategies by collecting human data (i.e. record actions of humans playing the game and measure their regulatory focus) and extract humans' strategies by using a classifier to determine the important features guiding their behaviors; 2/ designing "regulatory focus endowed" agents based on this classification, along with control agents "without personality"; 3 / test whether users perceive the intended agents' personalities in a controlled user study.

\section{A. Learning human strategies}

1) Data collection: Twelve dyads, composed of fifteen participants (13 men, 2 women ; age $\mathrm{M}=29,7$ years, SD $=10,2$ ), played Can't Stop games. Prior to the game session, each participant had answered the Regulatory Focus Questionnaire Proverbs Form (RFQ-PF), our own French questionnaire measuring the strengh of the two self-regulatory strategies with 18 questions to answer on a 7-point Likert scale. A psychometric study $(\mathrm{N}=277)$ validated the capacity of this questionnaire to measure chronic regulatory focus (in prep). Participants played via computers. To mimic a game with a distant opponent like an artificial agent, participants were seeing each other through webcams. They were allowed to talk to each other during the game.

2) Extracting strategies: To learn the behaviors, we trained two classifiers, one for each decision of the game: the choice of a movement and the stop-or-again decision. Features were related to the global state of the game (e.g. score difference at the time, numbers of playable columns), characteristics of candidate moves (e.g. distance from the top of a column, absolute and relative to the other possible moves) or statistics on the whole game (e.g. number of turns since the last loss). Both classifiers were binary classifiers. For the first classifier (movement decision), the target class was either "Selected" or "NotSelected", and the features considered two alternative moves, including the human selected move ${ }^{1}$. The dataset was composed of 1630 instances and 108 features. For the second classifier (stop-or-again), we considered one instance for each human player decision, with target class "Continue" if the player chose to continue and "Stop" otherwise. The dataset was composed of 670 instances and used 47 features (the number of features is higher for the first dataset since two alternative moves are considered).

3) Evaluating the classifiers: We choose to use the Alternative Decision Tree classifier (ADTree) [29] with 10 boosting iterations because the classifier (learned tree) is easy to interpret, which was required for our analysis to guide future symbolic implementation; the quality of the result was similar to other possible classifiers ${ }^{2}$. To determine the accuracy of the classifiers, we performed a 10 -fold cross-validation and benchmarked our results against different classifiers which

\footnotetext{
${ }^{1}$ For each human movement decision HD and each possible alternative AD, we added two instances, one with (HD,AD) and target="Selected" and one with $(\mathrm{AD}, \mathrm{HD})$ and target="NotSelected"

${ }^{2}$ We proceed to classification with Weka (version 3.7)
}

TABLE I

STATISTICS FROM THE 10-FOLD STRATIFIED CROSS-VALIDATION OF THREE DIFFERENT CLASSIFIERS ON PLAYING MODELS

\begin{tabular}{|c|c|c|c|}
\hline \multicolumn{4}{|c|}{ Movement choice model } \\
\hline & ZeroR & ADTree & RandomForest \\
\hline Incorrectly categorized items & $50,3 \%$ & $16,5 \%$ & $17,2 \%$ \\
\hline Kappa statistic & 0 & 0,67 & 0,66 \\
\hline ROC Area & 0,50 & 0,92 & 0,91 \\
\hline \multicolumn{4}{|c|}{ Stop or again decision model - without personality } \\
\hline & ZeroR & ADTree & RandomForest \\
\hline Incorrectly categorized items & $22,5 \%$ & $23,4 \%$ & $22,1 \%$ \\
\hline Kappa statistic & 0 & 0,25 & 0,22 \\
\hline ROC Area & 0,50 & 0,79 & 0,77 \\
\hline \multicolumn{4}{|c|}{ Stop or again decision model - with personality } \\
\hline & ZeroR & ADTree & RandomForest \\
\hline Incorrectly categorized items & $22,5 \%$ & $19.70 \%$ & $21,8 \%$ \\
\hline Kappa statistic & 0 & 0,33 & 0,23 \\
\hline ROC Area & 0,50 & 0,80 & 0,76 \\
\hline
\end{tabular}

finally presented equivalent performances compared to the ADTree classifiers.

As examples, we present in Table I the Zero-R (predicting the more frequent class, regardless of predictors; used as a baseline performance) the Random Forest (with 10 trees; known for good results with similar data) classifiers' performances. We looked at the number of incorrectly classified items, the kappa statistic and the receiver operating characteristic (ROC) area.

We observed that the ADTree's performances are sufficiently good compared to the other classifiers, and that the kappa statistic is at least fair for the three models. Thus, we considered the output of the ADTree classifier as consistent and coherent. For the movement choice model, personality scores were not selected as a feature in the tree. But personality scores were selected for the decision model and the classifier was more efficient when personality scores were taken into account. As an example, Figure 1 shows one branch of the ADT for the "stop-or-again" decision. At first, the number of throws in the turn is taken into account. If the player had already play one turn, then the quality of the position on the board is evaluated; else it depends on the promotion score.

\section{B. Designing the artificial agents}

In order to test the users' perception of agents' personality, we wanted a (scientifically speaking) control agent: an agent playing without personality. In psychology, there is no such thing as a person with no personality. So what could it be in affective computing? Two types of strategies are generally used as control in the domain: random strategy (but does the absence of planned consistency convey an absence of personality?) or "traditional AI" strategy (but does the absence of implemented personality is equivalent to the absence of personality?).

Thus we considered to have 4 types of agent:

- the random agent (Rand), which chooses randomly its moves and has a $50 \%$ probability to stop its turn;

- the "average" agent (Avg), which follows an ADTree 
Fig. 1. One branch of the Alternative Decision Tree for the decision model taking personality scores into account

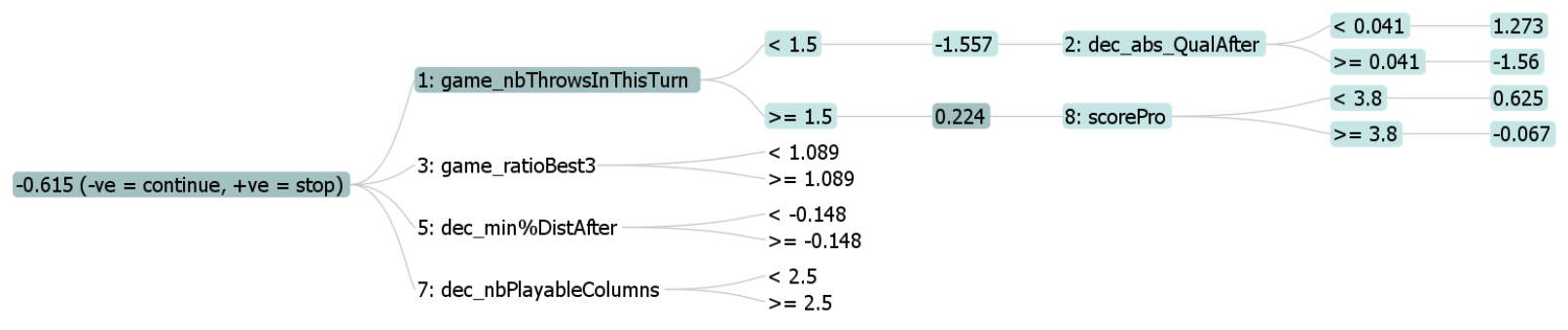

created without taking into account personality scores as a feature which should lead to a "depersonalized" strategy

- the promotion agent ( $R F$-Pro), which has a promotion score of 7 and a prevention score of 1 ;

- the prevention agent (RF-Pre), which has a promotion score of 1 and a prevention score of 7 . The so-called RFagents follow the same ADTree, where some branches are conditionned by the value of personality scores.

Users easily assign personality to things but, with the Randagent, the perceived pattern may be different for each user due to variability in behaviors while, with the Avg-agent, all users will be confronted with the same strategy, conveying a neither promotion nor prevention regulatory focus.

\section{Hypotheses}

Knowing the design of the artificial agents, we made 2 hypotheses concerning the results of the user study :

- H1: Differences in agents' personalities are perceived by the human player :

- H1a: For the rating of personality for the condition Rand, the interraters' agreement is low and the data dispersion is high.

- H1b: For the rating of personality for the condition Avg, the interraters' agreement is good and the data dispersion is low. The mean values of promotion and prevention scores are around the middle of the scale.

- H1c: For the rating of personality for the conditions $\mathrm{RF}$, the interraters' agreement is good and the data dispersion is low. The mean value of promotion score is on the upper part of the scale for the RF-Pro agent (and respectively for the prevention score concerning the RF-Pre agent).

- H2: The credibility of the agent is increased by the presence of personality. The RF-agents are perceived as more credible than the Rand-agent and the Avg-agent.

\section{User study}

1) Experimental design: Prior to the study, each subject had answered the RFQ-PF to assess their chronic regulatory focus.

During the study, the following procedure was applied. First, after signing a consent agreement, the participant was explained the rules of Can't Stop by viewing a video tutorial ${ }^{3}$. The possibility was given to the participant to pause the video and to go back if they needed to see again some part. Second, the participant played a tutorial game against the computer in order to become familiar with the game itself and its interface. The participant was informed that the computer would make random choices during the tutorial game. The experimenter answered the potential questions about Can't Stop. Third, the participant was informed that he or she would play 4 games against different artificial agents. The participant was also informed that he or she would have to evaluate the agent's personality after each game.

There was no visual display of the artificial agent, the only modality to evaluate the agent's personality was the way the agent played the game (see Figure 2). The different conditions were counterbalanced to compensate a potential effect of order. After each game against an agent, the participant answered the RFQ-PF in an other-ratings form (i.e. to characterize the agent's strategies during the game), along with 10 questions from the Godspeed Questionnaire [30]: 5 about likeability and 5 about the perceived intelligence of the agent (5-point Likert scale). We used these two scales as a credibility measure since perceived goodness and expertise are key dimensions of credibility [31]. We used a within-subject design in order for participants to have a "comparative" perception of agents' personalities. However, knowing that a game takes 15 to 20 minutes to play, participants only played one game with each agent to ensure a not too long experimental session. Because of the number of turns by game (20 on average), we thought that it would be sufficient for participants to make a personality judgment.

2) Participants: Twenty participants took part in this evaluation study. There were 11 men and 9 women (age $\mathrm{M}=30,6$ years, $\mathrm{SD}=8,1$ ). Of the participants, 17 were native french speakers and 3 were bilingual.

\section{RESULTS ${ }^{4}$ AND DISCUSSION}

First, we looked at the mean and standard deviation for each measure. We also computed the coefficient of quartile variation $(\mathrm{CQV} ;(Q 3-Q 1) /(Q 1+Q 3))$, which offers a comparable statistic of dispersion [32], and the Finn coefficient ${ }^{5}$ as an

\footnotetext{
${ }^{3}$ https://www.youtube.com/watch?v=kQs6JBX0txw

${ }^{4}$ Data were analysed using R, version 3.1.2, http://www.R-project.org

${ }^{5} \mathrm{R}$ package $i r r$, version 0.84
} 
Fig. 2. A user playing Can't Stop with an artificial agent during the agent's turn; board game as seen by the user (bottom left) and webcam record of the user's face (top right)

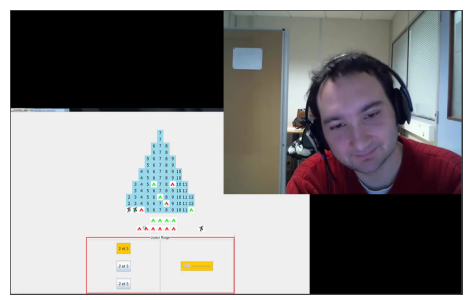

index of the interraters' agreement [33]. For interpretation of $\mathrm{CQV}$, dispersion of data is proportional to the percentage; for interpretation of the Finn coefficient, 0 means total disagreement and 1 means total agreement. Descriptive statistics are presented in Table II.

For the analysis of the differences between conditions, we applied non-parametric statistics, as the assumption of normality could not be granted in these conditions. We used the Friedman test as principal analysis and pairwise comparisons using Wilcoxon signed rank test as post-hoc test. For the post-hoc tests, p-values were adjusted using the Holm correction. Friedman tests reported significant differences for promotion-score $\left(\chi^{2}(3)=23,44 ; p<0,001\right)$, preventionscore $\left(\chi^{2}(3)=23,28 ; p<0,001\right)$ and the perceived intelligence $\left(\chi^{2}(3)=15,18 ; p=0,002\right)$. We do not found significant differences for the likeability $\left(\chi^{2}(3)=2,24 ; n . s\right.$. $)$. Post-hoc tests results are presented in Table III.

\section{A. Dispersion of data and interraters' agreement}

The CQV of the Rand agent for personality scores is, on average, 1.5 times higher than other agents' CQV. Finn coefficients of the Rand agent are the lowest for the promotion score and the prevention score (cf Table II).

This results validate our H1a hypothesis: participants have perceived a personality but not all the participants perceived the same personality.

\section{B. Personality scores}

For the promotion score, the RF-Pro agent and the Avgagent are rated high unlike the RF-Pre agent and the Randagent (cf Table II). Moreover, as shown in Table III, posthoc tests show that the RF-Pro agent is rated significantly higher than the RF-Pre agent and the Rand-agent. There is no significative difference between the RF-Pro agent and the Avgagent. Likewise, for the prevention score, the RF-Pre agent and the Rand-agent are rated high unlike the RF-Pro agent and the Avg-agent, as shown in Table II. The RF-Pre agent is rated significatively higher than the RF-Pro agent and the Avg-agent. There is no significative difference between the RF-Pre agent and the Rand-agent (cf Table III).

Our H1 hypothesis is partially validated: on the one hand, results show that our RF-Pro and RF-Pre agents have been respectively perceived as promotion-oriented and preventionoriented, as we expected (H1c validated). We could say that our data-driven strategies successfully convey the agent's regulatory focus. On the other hand, our Rand and Avg agents have been respectively perceived as prevention-oriented and promotion-oriented (H1b not validated).

This result points out the difficulty of controlling personality perception of virtual agents. As brought up by [34], by assessing personality with questionnaires, participants may be driven to rate something they had not perceived in the first place. Moreover, participants played only one game against each agent. The absence of repeated interactions prevents participants from perceiving the consistency in agents' strategies (or the lack of for Rand-agent). Nonetheless, the orientation of users' perception may be explained by looking at effective strategies used by the agents:

- The Rand-agent had a 50\% probability of stop its turn each time it had to choose. So, the probability $P$ of stopping after $i$ choices is $P_{i}=\frac{1}{2^{i}}$; short turns are more probable than long turns, thereby users have been more probably exposed to short turns. Short turns could be interpreted as a secure strategy, thus it is more probable that users rated the Rand-agent as prevention-focus.

- For the Avg-agent, we analyzed the learned tree to understand the promotion-focus orientation in users' perception. The tree shows a decision node on a feature describing the situation on the board, positive if my opponent is better placed than me on the board, negative else. If this feature is superior to 1 , a prediction node of value -1.61 is traversed (if the output of the tree is negative, agent choose to play again). So, as soon as the opponent has a better situation on the board than the agent's, there is a strong incentive to play again, promoting long turns which may have been interpreted as cue of a promotion-focus personality. Moreover, even if we have not the probabilities for each of the 81 possible outputs of the tree, only two of them lead the Avg-agent to stop its turn.

\section{Credibility scores}

For the likeability, scores are around 3 (on a 5-point scale) for all the agents (cf Table II). This can be the reflect of a light overall positive bias towards artificial agents, due to the person positivity bias (i.e. a natural tendency of the mind to focus on the optimistic at a subconscious level) [35]. The fact remains that we found no differences in likeability. Participants orally reported difficulties to evaluate likeability, because they found that the interaction was not sufficient to judge the agents' sympathy. Game strategies alone might not convey such a social concept. This result also raised another question: is selfreport sufficient to measure likeability, and if not, which kind of users' behaviors can be used to measure this concept?

For the perceived intelligence, scores (in Table II) have a higher range: from 2,63 (Rand-agent) to 3,85 (RF-Pre agent). The RF-Pre agent is rated significantly higher than the others. Although the RF-Pro agent was rated as more intelligent than the Rand and Avg agents, there is no statistically significant 
TABLE II

DESCRIPTIVE STATISTICS OF THE DIFFERENT SCORES COLLECTED DURING THE STUDY

Pro Sc. = promotion score $(\min =1 ; \max =7) ;$ Pre Sc. = prevention score $(\min =1 ; \max =7) ;$ Lik. $=$ likeability $(\min =1 ; \max =5) ;$ Perc. Int.$=$ perceived intelligence ( $\min =1 ; \max =5) ; C Q V=$ Coefficient of Quartile Variation

\begin{tabular}{clcccc}
\hline & & Rand & Avg & RF-Pro & RF-Pre \\
\hline \multirow{5}{*}{ Pro Sc. } & Mean & 3,53 & 5,3 & 5,26 & 3,09 \\
& SD & 1,47 & 1,44 & 1,28 & 1,22 \\
& FQV & $32 \%$ & $15 \%$ & $14 \%$ & $20 \%$ \\
& Finn coeff. & 0,46 & 0,48 & 0,59 & 0,63 \\
\hline \multirow{4}{*}{ Pre Sc. } & Mean & 4,51 & 3,18 & 2,91 & 5,58 \\
& SD & 1,71 & 1,4 & 1,33 & 0,73 \\
& CQV & $30 \%$ & $37 \%$ & $25 \%$ & $8 \%$ \\
& Finn coeff. & 0,27 & 0,51 & 0,56 & 0,87 \\
\hline \multirow{4}{*}{ Lik. } & Mean & 3,3 & 3,22 & 3,02 & 3,51 \\
& SD & 0,78 & 0,67 & 0,95 & 0,75 \\
& CQV & $19 \%$ & $13 \%$ & $18 \%$ & $14 \%$ \\
\hline \multirow{4}{*}{ Perc. Int. } & Mean & 2,63 & 2,94 & 3,11 & 3,85 \\
& SD & 0,6 & 1,16 & 0,98 & 0,68 \\
& CQV & $18 \%$ & $33 \%$ & $27 \%$ & $11 \%$ \\
\hline
\end{tabular}

TABLE III

P-VALUES OF THE POST-HOC TESTS FOR THE PROMOTION SCORE, THE PREVENTION SCORE AND THE PERCEIVED INTELLIGENCE $* p \leq 0.05 ; * * p \leq 0.01 ; * * * p \leq 0.001$

\begin{tabular}{llll}
\hline \multicolumn{4}{c}{ Promotion score } \\
\hline Avg & Rand & Avg & RF-Pro \\
RF-Pro & 0,07 & - & - \\
RF-Pre & $0,03^{*}$ & 0,42 & - \\
\hline \multicolumn{4}{c}{ Prevention score } \\
\hline \multicolumn{5}{c}{ Rand } & Avg & RF-Pro \\
Avg & $0,01 *$ & - & - \\
RF-Pro & $0,004^{* *}$ & 1,00 & - \\
RF-Pre & 0,51 & $0,005^{* *}$ & $0,001^{* * *}$ \\
\hline \multicolumn{5}{c}{ Perceived intelligence } \\
\hline Avg & Rand & Avg & RF-Pro \\
RF-Pro & 0,63 & - & - \\
RF-Pre & $0,002^{* *}$ & 0,79 & - \\
\hline
\end{tabular}

difference between the three agents (cf Table III). Considering our number of subjects, we could not say if the nonsignificance is due to a lack of data or to a real difference due to the agent's strategy. We noted that the RF-Pre agent won $47 \%$ of its games, while it goes from $42 \%$ for the RFPro agent, to $32 \%$ for the Avg-agent and down to 5\% for the Rand-agent.

\section{CONCLUSION AND PERSPECTIVES}

To conclude, we have shown that it is possible to successfully endow artificial agents with regulatory focus with a data-driven approach. Moreover, the implemented regulatory focus can be accurately perceived by users. We believe that regulatory focus could be of use for affective and persuasive computing. Indeed, regulatory focus theory comes with the concept of regulatory fit. Regulatory fit states that matching user's regulatory-focus and means used to approach one goal creates a feeling of rightness about the pursued goal and increases task engagement [36]. For example, a user in a state of promotion-focus will be more receptive to promotionoriented messages (and respectively for prevention-focus) [37]. For artificial companions (trying to build a relationship with their users) or persuasive technologies (aiming to change behaviors of users through social influence), creating a situation of regulatory fit could be a good place to start.

As we advocate for a mutually beneficial personality science for psychologists and for computer scientists, we believe that producing artificial behaviors that will put a personality across via data-driven techniques is only a first step. The next step will be to generalize the knowledge available in the learned trees in order to implement a symbolic goal-based architecture for Can't Stop artificial players and see if we could replicate our data-driven results. Indeed, we think of data-driven and theory-driven approaches as complementary: the former will feed the latter. One theory-driven symbolic implementation will computationally approach cognitive processes and provide a clearer view of parameters accounting for personality in these processes. By making a bridge between data-driven results and theory-driven implementation, we hope to provide valuable and interpretable cognitive simulations that could be used by psychologists to evaluate and enrich their models.

Besides, we raised methodological concerns about experimental testing of concepts such as personality or likeability in computer sciences (e.g. how to assess them; how to have a good control condition). As long as personality perception is concerned, we stress the temporal issue of repetition. We believe repeated interactions are necessary to go from impression formation to a deeper personality judgment.

As perspectives, we list directions for future works in order to try to provide data for answering the questions raised by our results and better understand the functioning of personality with artificial agents:

- making more longitudinal studies because only repeated interactions could allow users to form a real model of the agent's personality;

- complementing self-report measures by users' behaviors measures, such as engagement for example;

- investigating if personality has other impacts on the game using subjective and objective measures (e.g. game outcome, user's strategy);

- implementing an "optimally-playing" agent as another control;

- using multi-modality to enhance the interaction, such as verbal and non-verbal behaviors during the game by providing a physical representation of a virtual agent.

\section{ACKNOWLEDGMENTS}

This work is conducted as a part of the French funded ANR My Little Artificial Companions World - MOCA project ANR-2012-CORD-019-02.

\section{REFERENCES}

[1] D. Benyon and O. Mival, "From human-computer interactions to humancompanion relationships," in Proceedings of the First International 
Conference on Intelligent Interactive Technologies and Multimedia, 2010.

[2] D. Sviatlana, S. Busemann, and C. Schommer, "Artificial conversational companions: A requirements analysis," in Proceedings of the 4th International Conference on Agents and Artificial Intelligence, Vilamoura, Portugal, 2012, pp. 282-289.

[3] W. Revelle and K. R. Scherer, "Personality and emotion," Oxford companion to emotion and the affective sciences, pp. 304-306, 2009.

[4] J. K. Burgoon, J. A. Bonito, B. Bengtsson, C. Cederberg, M. Lundeberg, and L. Allspach, "Interactivity in humancomputer interaction: A study of credibility, understanding, and influence," Computers in Human Behavior, vol. 16, no. 6, pp. 553-574, 2000.

[5] K. Isbister and P. Doyle, "Design and evaluation of embodied conversational agents: A proposed taxonomy," in The First International Joint Conference on Autonomous Agents \& Multi-Agent Systems, 2002.

[6] R. Niewiadomski, V. Demeure, and C. Pelachaud, "Warmth, competence, believability and virtual agents," in Proceedings of the 10th International Conference on Intelligent Virtual Agents, 2010, pp. 272 285.

[7] A. Vinciarelli and G. Mohammadi, "More personality in personality computing," IEEE Transactions on Affective Computing, vol. 5, no. 3, pp. 291-300, 2014

[8] B. J. Scholl and P. D. Tremoulet, "Perceptual causality and animacy," Trends in Cognitive Sciences, vol. 4, no. 8, pp. 299-309, 2000.

[9] J. A. Benfield, W. J. Szlemko, and P. A. Bell, "Driver personality and anthropomorphic attributions of vehicle personality relate to reported aggressive driving tendencies," Personality and Individual Differences, vol. 42, no. 2, pp. 247-258, 2007.

[10] C. Nass and Y. Moon, "Machines and mindlessness: Social responses to computers," Journal of Social Issues, vol. 56, no. 1, pp. 81-103, 2000.

[11] P. J. Gmytrasiewicz and C. L. Lisetti, "Emotions and personality in agent design and modeling," in Game theory and decision theory in agent-based systems. Springer, 2002, pp. 81-95.

[12] P. T. Costa and R. R. McCrae, "Four ways five factors are basic," Personality and Individual Differences, vol. 13, no. 6, pp. 653-665, 1992.

[13] A. Vinciarelli and G. Mohammadi, "A survey of personality computing," IEEE Transactions on Affective Computing, vol. 5, no. 3, pp. 273-291, 2014.

[14] A. O. J. Cramer, S. Sluis, A. Noordhof, M. Wichers, N. Geschwind, S. H. Aggen, K. S. Kendler, and D. Borsboom, "Dimensions of normal personality as networks in search of equilibrium: You can't like parties if you don't like people," European Journal of Personality, vol. 26, no. 4, pp. 414-431, 2012.

[15] A. Bandura, "Social cognitive theory of personality," in Handbook of personality, 2nd ed., L. Pervin and O. John, Eds. New York: Guilford Publications, 1999, pp. 154-196.

[16] W. Mischel, Y. Shoda, and R. Smith, Introduction to Personality: Towards an Integration, 7th ed. John Wiley \& Sons, 2004.

[17] M. Poznanski and P. Thagard, "Changing personalities: towards realistic virtual characters," Journal of Experimental \& Theoretical Artificial Intelligence, vol. 17, no. 3, pp. 221-241, 2005.

[18] S. J. Read, B. Monroe, A. Brownstein, Y. Yang, G. Chopra, and L. C. Miller, "A neural network model of the structure and dynamics of human personality," Psychological Review, vol. 117, no. 1, pp. 61-92, 2010.

[19] T. Rothmund, A. Baumert, and M. Schmitt, "Can network models represent personality structure and processes better than trait models do?" European Journal of Personality, vol. 26, no. 4, pp. 444-445, 2012.

[20] C. Faur, C. Clavel, S. Pesty, and J.-C. Martin, "Perseed: a self-based model of personality for virtual agents inspired by socio-cognitive theories," in Proceedings of Affective Computing and Intelligent Interaction (ACII 2013). Humaine Association Conference on., Geneva, Swiss, 2013, pp. 467-472.

[21] E. T. Higgins, "Beyond pleasure and pain." American psychologist, vol. 52, no. 12, pp. 1280-1300, 1997.

[22] R. Bartle, "Hearts, clubs, diamonds, spades: Players who suit MUDs," Journal of MUD research, vol. 1, no. 1, 1996

[23] D. Johnson and J. Gardner, "Personality, motivation and video games," in 22nd Conference of the Computer-Human Interaction Special Interest Group of Australia on Computer-Human Interaction. Queensland University of Technology, Brisbane, QLD: ACM Press, 2010, pp. 276281.

[24] C. Elliott, "Research problems in the use of as shallow artificial intelligence model of personality and emotion," in Proceedings of the 12th national conference on Artificial intelligence (AAAI'94), vol. 1. American Association for Artificial Intelligence., 1994, pp. 9-15.

[25] M. Johansson and H. Verhagen, "Social believability in games - the early years," in Proceedings of Social Believability in Games Workshop, Colocated with the 9th International Conference on the Foundations of Digital Games (FDG2014), 2014.

[26] J. Gratch, S. Marsella, N. Wang, and B. Stankovic, "Assessing the validity of appraisal-based models of emotion." in Proceedings of the 3rd International Conference on Affective Computing and Intelligent Interaction and Workshops (ACII 2009), 2009, pp. 147-154.

[27] M. Courgeon, C. Clavel, and J.-C. Martin, "Modeling facial signs of appraisal during interaction: impact on users' perception and behavior," in Proceedings of the 2014 international conference on Autonomous agents and multi-agent systems. International Foundation for Autonomous Agents and Multiagent Systems, 2014, pp. 765-772.

[28] Wikipedia. (2015) Can't stop (board game) - Wikipedia, the free encyclopedia. [Online]. Available: https://en.wikipedia.org/w/index.php? title $=$ Can \%27t_Stop_\%28board_game\%29\&oldid $=591302325$

[29] G. Holmes, B. Pfahringer, R. Kirkby, E. Frank, and M. Hall, "Multiclass alternating decision trees," in Machine learning: ECML 2002. Springer, 2002, pp. 161-172.

[30] C. Bartneck, D. Kuli, E. Croft, and S. Zoghbi, "Measurement instruments for the anthropomorphism, animacy, likeability, perceived intelligence, and perceived safety of robots," International Journal of Social Robotics, vol. 1, no. 1, pp. 71-81, 2009.

[31] S. Tseng and B. Fogg, "Credibility and computing technology," Communications of the ACM, vol. 42, no. 5, pp. 39-44, 1999.

[32] D. G. Bonett, "Confidence interval for a coefficient of quartile variation," Computational Statistics \& Data Analysis, vol. 50, no. 11, pp. 29532957, Jul. 2006.

[33] R. H. Finn, "A note on estimating the reliability of categorical data." Educational and Psychological Measurement, 1970.

[34] K. Liu, J. Tolins, J. E. F. Tree, M. Walker, and M. Neff, "Judging IVA personality using an open-ended question," in Intelligent Virtual Agents. Springer, 2013, pp. 396-405.

[35] D. O. Sears, "The person-positivity bias." Journal of Personality and Social Psychology, vol. 44, no. 2, pp. 233-250, 1983.

[36] E. T. Higgins, "Value from regulatory fit," Current Directions in Psychological Science, vol. 14, no. 4, pp. 209-213, 2005.

[37] A. Y. Lee and J. L. Aaker, "Bringing the frame into focus: The influence of regulatory fit on processing fluency and persuasion." Journal of Personality and Social Psychology, vol. 86, no. 2, pp. 205-218, 2004. 